\title{
Measurement of the Nonuniformity of First Responder Thermal Imaging Cameras
}

\author{
ANDREW LOCK and FRANCINE AMON \\ Fire Research Division \\ National Institute of Standards and Technology \\ 100 Bureau Drive (Stop 8663) \\ Gaithersburg, MD 20899-8663, USA
}

\section{ABSTRACT}

Police, firefighters, and emergency medical personnel are first responders that use thermal imaging cameras (TICs) every day. However, few performance metrics have been developed to assist first responders in evaluating thermal imaging technology. This paper describes a metric for evaluating the nonuniformity of commercial TICs. Three TICs, each employing a different thermal detection technology, were examined. Each TIC was considered a 'black box' while being tested. An extended area blackbody (178 mm square) was used as the thermal target, and was placed very close to the objective lens of the TIC under test. The resulting National Television Systems Committee (NTSC) video output signal of the TICs was collected, digitized, and processed. The nonuniformity was calculated using the standard deviation of the digitized image pixel intensities divided by the mean of those pixel intensities. This procedure was repeated for each TIC at several blackbody temperatures in the range from $30^{\circ} \mathrm{C}$ to $260{ }^{\circ} \mathrm{C}$. It was observed that the nonuniformity increased in a roughly linear manner with temperature. The testing procedure described herein was developed as part of a suite of tests to be incorporated into a performance standard addressing TICs used by the fire service.

KEYWORDS: evaluation, first responder, infrared, nonuniformity, performance metrics, test methods, thermal imaging camera

\section{NOMENCLATURE LISTING}

$\begin{array}{llll}N U & \text { nonuniformity } & \sigma & \text { standard deviation } \\ m & \text { mean } & y_{i j} & \text { output of nonuniformity correction } \\ x_{i j} & \text { input of nonuniformity correction } & G_{i j} & \text { gain of an individual pixel } \\ O_{i j} & \text { offset of an individual pixel } & & \end{array}$

\section{INTRODUCTION}

Thermal imaging cameras (TICs) are becoming an important tool for many firefighters and other first responders. Progress in technology and manufacturing in recent years has developed to such a level that TICs are affordable enough for most fire departments to purchase and are therefore receiving more widespread use throughout the country. However, due to the lack of performance standards for TICs, a wide variety of designs and capabilities are provided to end users with little consistency in reported performance. Therefore, the National Fire Protection Association (NFPA) is developing a new performance standard, NFPA 1801 [1], for TIC used by the fire service in order to ensure the appropriate quality and capabilities for these tools to be used in fire applications.. Working toward that goal, the National Institute of Standards and Technology (NIST) has been conducting research into the performance of TICs with a special emphasis on the TIC's image quality.

There are currently three well-established detector/sensor technologies available for commercial purchase: barium-strontium-titanate (BST), vanadium oxide (VOx), and amorphous silicon (ASi). While the thermal detectors used in fire service applications are uncooled focal plane arrays (FPA), utilizing an array of sensors located at the focal plane of the optics, each specific detector technology is capable of generating somewhat different levels of information in the displayed image. The two typical FPA sensor configurations are 240 rows x 320 columns and 120 rows x 160 columns. The BST detectors are solid-state ceramic devices that convert changes in electrical polarization to voltage differences at nodes on the 
detector surface. A thermoelectric cooler provides thermal stability. These are AC coupled detectors that measure relative levels of infrared radiation, thus the detector output requires a correction based on reference points continually provided by a chopper. The VOx and ASi detectors are both microbolometer devices, meaning that the detectors are actually comprised of an array of very small individual detectors (often referred to as pixels). Microbolometers measure changes in the electrical resistance of the sensor material due to heat from infrared radiation; they don't require cooling but are relatively sensitive to the temperature of the detector array. A shutter is used to periodically reset the detector's absolute radiation input level. When the incident infrared radiation becomes so high that it saturates a predetermined percentage of the pixels in the detector array, the integration (exposure) time of the detector is automatically reduced. This mechanism, referred to as mode shift, reduces the thermal sensitivity of the detector but allows detection of a larger range of surface temperatures. Note that mode shifting does not apply to BST detectors.

The signal from the FPA is relayed to the imager's signal processor via a charge-coupled device. The optics package provides an interface between the signal processor and the recorded image and makes an important contribution to the overall performance of the instrument. Many factors can influence the TIC's image quality, including the spectral response of the thermal imaging core, the number of pixels in the FPA, the quality of the optical components, the specific IR sensing technology employed, and the internal image processing conducted by the TIC to produce the best possible image. There are as many ways of designing optics and electronics packages as there are TIC form factors and target market prices. The test method discussed in this work, along with the other test methods developed for the NFPA standard, is designed to treat the TIC as a 'black box' in which the performance of the various components is not considered. The overall performance of the TIC as a complete system is of primary concern.

Nonuniformity is inherent to all TICs, due mostly to the difficulty of producing FPAs that are perfectly uniform with nodes or pixels that have the same linear response [2]. Therefore, TICs incorporate an automatic nonuniformity correction (NUC) into their design. As mentioned previously, the BST detectors use a chopper to provide reference points for the NUC, while microbolometers use a shutter. The specific algorithms for NUC are proprietary, therefore this discussion will be general. A simple and common NUC is accomplished by use of a 2-point correction [3-5]. The 2-point NUC assumes that each node or pixel, $i, j$, in the detector has a linear response to signal input as shown in equation (1):

$$
y_{i j}=G_{i j} \cdot x_{i j}(\phi)+O_{i j}
$$

Here, $x_{i j}$ is the input signal, $G_{i j}$ is the gain, $O_{i j}$ is the offset, and $y_{i j}$ is the corrected output. Upon the assumption of a linear detector response, the FPA is calibrated by making measurements at two points: a low temperature and a high temperature, in order to determine the gain and offset parameters. It is also assumed that the gain is preset and does not change. TICs automatically conduct a recalibration of the offset by continually monitoring the chopper or periodically closing the shutter for a brief time, assuming the chopper or shutter is at room temperature.

The 2-point NUC is unable to correct many of the nonuniformities discussed in this paper because the detector response is not linear as the 2-point model assumes, although the response can be approximated as linear for a small temperature range. The calibration is conducted such that the nonuniformity is corrected at room temperature. Therefore, within a small variation of temperature from room temperature very little change in nonuniformity is observed. However, as the uniform temperatures are increased, the nonlinearity in detector response becomes more apparent, since the gain of each pixel is maintained constant, and larger nonuniformity is measured. There are other more sophisticated approaches to NUC, including adaptive scene based approaches and neural networks [4-7]. Many of these methods are too sophisticated and slow for implementation in handheld, uncooled TICs used by the fire service.

This paper presents a practical methodology for measuring the nonuniformity of TICs used by the fire service. Three TICs, each representing one of the three different detector technologies are tested by this method and the results are discussed and compared. The variation of the nonuniformity of each TIC with temperature is examined and the effect of mode shifts is considered as well. 


\section{METHODOLOGY}

Nonuniformity is a measure of “...large area blemishes, blotches, and shading effects that may be distracting to the observer..." [2]. Nonuniformity refers to the existence of blemishes, whereas uniformity is the opposite; these terms are often used interchangeably in literature. Image nonuniformities can be the result of either spatial or temporal variations in the array. In fact some researchers disagree about the specific definition of "Nonuniformity" and advocate separate isolation of temporal and spatial variation in the camera's output [7]. The definition of nonuniformity presented by Holst will be utilized in this report [2]. Specifically, spatial variations are primarily analyzed and temporal variations are minimized by averaging multiple images collected over time.

Generally, nonuniformity is measured using a uniform prescribed intensity (in this case an extended area blackbody source), which entirely fills the field of view of the camera under test. The uniform intensity field is imaged and recorded, and the statistical mean, $m$, and standard deviation, $\sigma$, of the image pixel intensities are calculated. The nonuniformity, $N U$, is then given by the ratio of the standard deviation to the statistical mean, which effectively makes the $N U$ independent of system gain [2]. The calculations presented here are reported based on equation (2):

$$
N U=\frac{\sigma}{m}
$$

A CI Systems SR80-HT (Certain companies and commercial properties are identified in this paper in order to specify adequately the source of information or of equipment used. Such identification does not imply endorsement or recommendation by the National Institute of Standards and Technology, nor does it imply that this source or equipment is the best available for the purpose.) $178 \mathrm{~mm}$ square extended area blackbody was used as a target for the nonuniformity measurements presented in this investigation. This blackbody has a range of $30{ }^{\circ} \mathrm{C}$ to $450{ }^{\circ} \mathrm{C}$, is accurate to within $0.5{ }^{\circ} \mathrm{C}$, and has a stability of $0.15{ }^{\circ} \mathrm{C}$ according to the instrument documentation. The blackbody surface temperature accuracy and nonuniformity was measured independently at the Physics Laboratory at NIST using a Raytek TRT LT 5C703 (spectral band $8 \mu \mathrm{m}$ to $14 \mu \mathrm{m}$, nominal spot size $5 \mathrm{~mm}$ ) transfer standard pyrometer. The uncertainty in the temperature measurements is $0.1{ }^{\circ} \mathrm{C}$ (coverage factor of $\mathrm{k}=2$ ). Minimizing the spatial nonuniformity of the blackbody surface ensures that the nonuniformities being measured are in fact inherent to the TIC and not originating in the source. The nonuniformity of the blackbody was measured at the same set-point temperatures used for the measurement of TIC nonuniformity: $30^{\circ} \mathrm{C}, 100{ }^{\circ} \mathrm{C}, 200{ }^{\circ} \mathrm{C}$, and $260^{\circ} \mathrm{C}$. Figure 1 shows a contour map of the surface temperature measurements of the blackbody at a set point of $100{ }^{\circ} \mathrm{C}$. The difference between the set-point and the measured mean temperature of the blackbody surface is consistently $3 \%$ and is attributed to differences in the measuring technique between the blackbody temperature control and the transfer standard pyrometer as well as a deviation in the actual surface emissivity from the documented value. The variation in the uniformity of the blackbody surface is $0.5^{\circ} \mathrm{C}$. This corresponds to a blackbody surface temperature nonuniformity of $N U=0.01$ at $100{ }^{\circ} \mathrm{C}$, which is much smaller than the nonuniformities being measured from the TICs. In fact, for all of the temperatures for which the blackbody nonuniformity was tested, the nonuniformity of the blackbody surface was never more than $N U=0.01$.

The blackbody was intentionally placed far out of the focal plane of the TIC, $5 \mathrm{~cm}$ from the TIC lens, as shown in Fig. 2. The out-of-focus blackbody approach was used because it is very difficult to produce a focused, uniform surface temperature of the size necessary for this application [6, 7], which, considering the wide field of view $\left(45^{\circ}\right.$ to $\left.60^{\circ}\right)$ and $1 \mathrm{~m}$ minimum focusing distance of most fire service TICs, would be at least $1 \mathrm{~m}$ by $1 \mathrm{~m}$ square. The out-of-focus blackbody approach works on the assumption that the blackbody is a Lambertian surface, i.e., its thermal radiance is constant when viewed from any angle. Placing a fire service TIC within $5 \mathrm{~cm}$ of a blackbody face that is much larger than the TIC's field of view is the optical equivalent of placing the TIC inside an integrating sphere of infinite diameter. Additionally, any nonuniformity that may exist on the blackbody surface will be sufficiently defocused so that its effect is distributed over a large portion of the TIC detector.

The FPA configuration of the TICs used in this work were 120 rows by 160 columns for both microbolometers (VOx TIC and ASi TIC), and 240 rows by 320 columns for the BST TIC. 


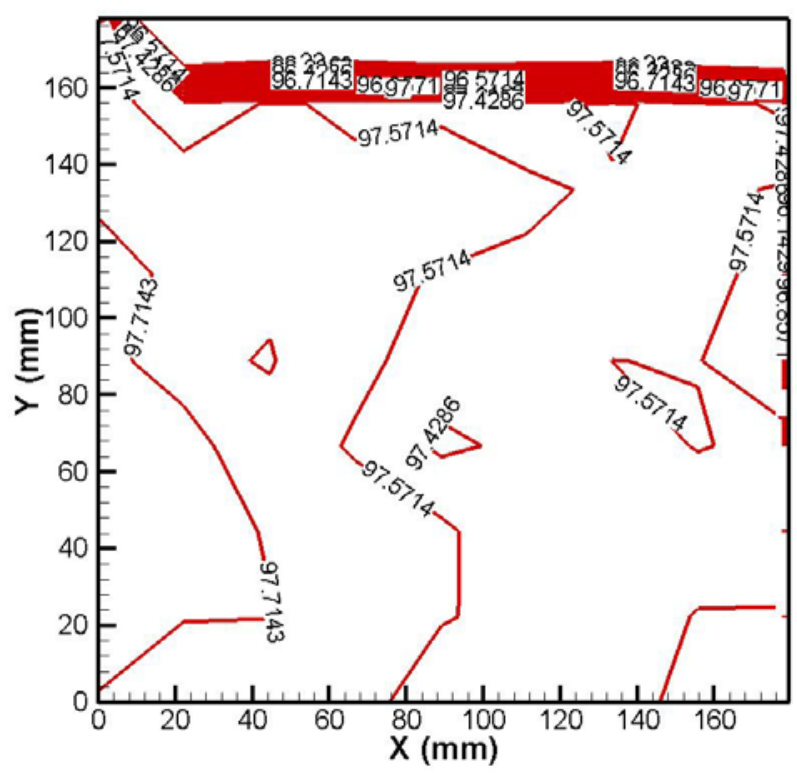

Fig. 1. Contour map of the thermal nonuniformity of the $178 \mathrm{~mm}$ blackbody surface at a nominal temperature of $100{ }^{\circ} \mathrm{C}$. The maximum difference in temperature on the surface of the blackbody was $0.5^{\circ} \mathrm{C}$ which corresponds to $N U=0.01$ for the blackbody surface. The edge effects on the far right of the image are due to a misalignment of the pyrometer and not indicative of the blackbody surface nonuniformity.

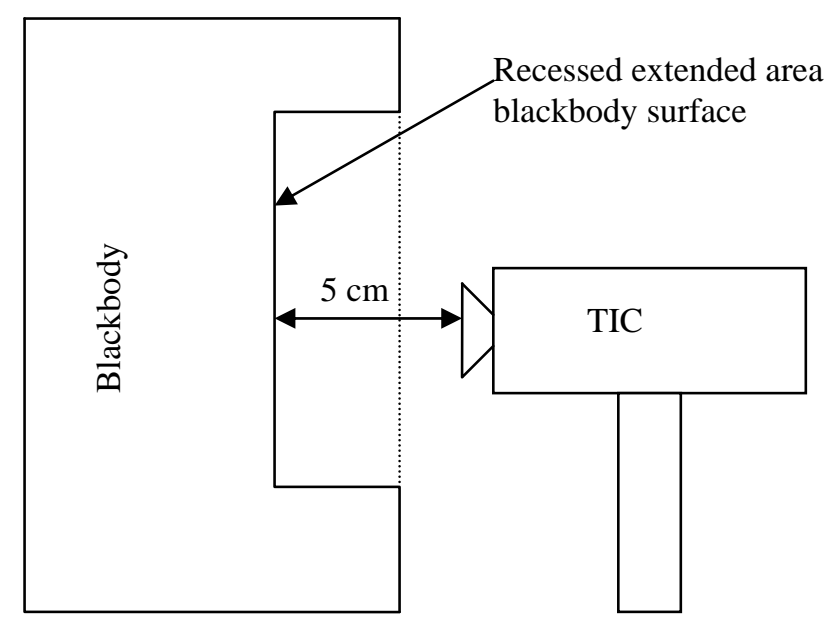

Fig. 2. Positioning of TIC for nonuniformity measurement with $178 \mathrm{~mm}$ extended area blackbody. The TIC is positioned so that the blackbody completely fills the cameras field of view. Generally, the entire blackbody surface is not imaged. 


\section{Data Collection}

The tests were conducted in a space that nominally meets standard temperature and pressure conditions $\left(20^{\circ} \mathrm{C}\right.$ and $\left.1 \mathrm{~atm}\right)$. No large sources of heat or high air flowrates that might influence the test results were present in the immediate vicinity of the testing station. Large heat sources can create reflections on surfaces, which may interfere with results. Large flowrates of air can exacerbate the effect of density gradients in the field of view of the TIC. Flow of air may also make it difficult to maintain a uniform blackbody surface temperature during testing due to convective heat losses. Temperature set-points of $30^{\circ} \mathrm{C}, 100{ }^{\circ} \mathrm{C}, 200{ }^{\circ} \mathrm{C}$, and $260{ }^{\circ} \mathrm{C}$ were chosen because they represent human body temperature, water boiling temperature, a convenient mid-range temperature, and the temperature at which certain other firefighter equipment is tested, respectively [8]. Prior to beginning each test, the blackbody was allowed to reach a steady state at the prescribed temperature. The TIC was turned on for 5 min before each test and only placed in front of the black body for less than a minute while the test was executed. The TIC was mounted in the nominal working position with TIC line of sight positioned horizontally and normal to the blackbody surface, as shown in Fig. 2. The TIC lens was positioned so that it was $5 \mathrm{~cm}$ away from the blackbody surface such that its entire field of view was encompassed by the blackbody. Each TIC was outfitted with a video output cable that was connected to a National Instruments digital video recording device. The digital video recording device records $10 \mathrm{~s}$ of NTSC standard video with a bit depth resolution of 8 bits at 30 frames per second.

The simplest analysis of the nonuniformity is accomplished by implementing equation (2) to determine $N U$. Each frame in the series of video images is analyzed to determine a statistical mean and standard deviation, which are then averaged over the test duration. The average $N U$ value of the image series is then reported. The standard deviation of $N U$ over all of the images in the test sequence is reported as the uncertainty in the measurement (with a coverage factor of 2 and represented by error bars in plots of nonuniformity). Two additional issues must be taken into account in order to perform these calculations. First, any extraneous TIC display characters, icons, or symbols must be removed from the each of the images. These items are present in most TIC images as enunciators for the status of the TIC, e.g., temperature readout, overheat indicator, remaining battery life, etc... If the test is being done to evaluate the TIC as observed by a user, a display correction factor should be applied which correlates the video signal from the TIC video output to the image presented on the TIC display [9].

\section{RESULTS AND DISCUSSION}

Before discussing the quantitative test results, it is instructive to observe the qualitative variations in image nonuniformity. In Fig. 3-6, representative images of pixel intensity captured from each TIC at temperatures of $30^{\circ} \mathrm{C}, 100{ }^{\circ} \mathrm{C}, 200{ }^{\circ} \mathrm{C}$, and $260{ }^{\circ} \mathrm{C}$, respectively, are presented. Starting with Fig. 3 at $30^{\circ} \mathrm{C}$, all TICs provide a relatively uniform image (low nonuniformity). In Fig. 4 at $100{ }^{\circ} \mathrm{C}$, several types of nonuniformities begin to appear. In the BST TIC image, there are two dominate nonuniformities present: a narcissus effect, the camera seeing its own optics, is observed as the faint circle in the center of the image; and diagonal shading becomes apparent, which is an artifact of the chopper built into the BST TIC. The VOx TIC presents a different type of nonuniformity. The TIC still has a largely uniform display image at this temperature, however the vertical lines of pixels are becoming visible due to the non-uniform response of each column and the fact that this difference is maximized near the microbolometer's pixel saturation limit, far from the temperature at which the nonuniformity correction is applied. The ASi TIC shows combinations of the types of nonuniformities observed in the BST and VOx TICs above, but at higher levels. Narcissus, pixel saturation and inherent defects in the optics and/or detector are apparent (diagnosis of the source of the defects is uncertain without disassembly of the TIC).

Figure 5 presents characteristic images from each of the TICs taken at $200^{\circ} \mathrm{C}$. The nonuniformities in the BST TIC that were noticed in Fig. 4 are becoming more profound. The narcissus does not appear to be much worse, but the diagonal shading has become quite significant. Recall that TIC employing BST detectors do not have a mode shift mechanism, therefore the images produced by these detectors tend to deteriorate as the target temperature increases to a maximum tolerable level. For the VOx TIC, the image now appears to be quite uniform, this is primarily due to the fact that with the increased temperature the camera has shifted mode to a lower thermal sensitivity so nonuniformities would inherently be smaller. The ASi TIC has shifted mode as well, this TIC appears to be more uniform than at $100{ }^{\circ} \mathrm{C}$, however, other 
than pixel saturation, most of the ASi TIC nonuniformities are still present. Figure 6 presents characteristic images from each of the TICs recorded at $260^{\circ} \mathrm{C}$. Here we see the largest nonuniformities in the BST TIC, which is related to the diagonal shading of the image and the absence of a mode shift mechanism. There are no significant observable differences in the relative image nonuniformity of the VOx TIC based on the images presented here. With the ASi TIC, a starker contrast is apparent at $260^{\circ} \mathrm{C}$ than at $200{ }^{\circ} \mathrm{C}$, and there is a noticeable increase in nonuniformity at $260^{\circ} \mathrm{C}$.

The general observation that can be made based on Fig.s 3-6 is that the nonuniformity of each TIC increases with increasing target temperature, except at the mode shift in the VOx and ASi systems. When the sensitivity of the microbolometer systems is decreased due to the mode shift there is a corresponding decrease in nonuniformity. This suggests that the nonuniformity is scaling with temperature, but on a fundamental level it is actually scaling with pixel saturation.

The variation of $N U$ with temperature is also plotted in Fig. 7. The same trends discussed above are apparent in the quantitative results and will be discussed with respect to the $N U$ dependence on target temperature. In order to get a better idea of the relationship between $N U$ and target temperature, additional temperature points are included for $\mathrm{T}=65^{\circ} \mathrm{C}, 150^{\circ} \mathrm{C}$, and $230^{\circ} \mathrm{C}$.

The BST TIC $N U$ trend is represented by the solid line in Fig. 7. It exhibits a roughly linear increase in nonuniformity with temperature. There is a monotonous increas in $N U$ for this TIC because there is no mode shift in the BST architecture. The VOx TIC $N U$ trend is represented by the dashed line; this TIC consistently exhibited the lowest $N U$ values across the entire target temperature range. The lowest nonuniformity occurs at $30^{\circ} \mathrm{C}$ and $N U$ increases roughly linearly to $100{ }^{\circ} \mathrm{C}$. Between $100{ }^{\circ} \mathrm{C}$ and $150{ }^{\circ} \mathrm{C}$ the nonuniformity begins to decreases due to internal image enhancement done by the camera as evidenced by large uncertainties in the measurement due to the camera continually trying to improve the image. The $N U$ reaches a second minimum at $230^{\circ} \mathrm{C}$ due to the mode shift in the camera occurring at this temperature. Then, between $230{ }^{\circ} \mathrm{C}$ and $260{ }^{\circ} \mathrm{C}$ the nonuniformity increases linearly again, to peak at $260{ }^{\circ} \mathrm{C}$. This observation is only valid for the particular TIC being tested, other VOx TICs may reach a maximum $N U$ prior to the mode shift. The slope of $N U$ prior to the mode shift is nearly identical to that after the mode shift for the VOx TIC. The ASi TIC $N U$ trends are represented by the dotted line in Fig. 7. The trends for the ASi TIC are qualitatively similar to those observed for the VOx TIC. Two differences are that the VOx TIC has a consistently lower $N U$ relative to the ASi TIC and the post mode shift $N U$ low point occurs at different target temperatures. The ASi TIC exhibits a maximum $N U$ at $100^{\circ} \mathrm{C}$, similar to that observed for the VOx TIC. As with the VOx TIC, the ASi TIC NU increases at similar slopes before and after the mode shift. 

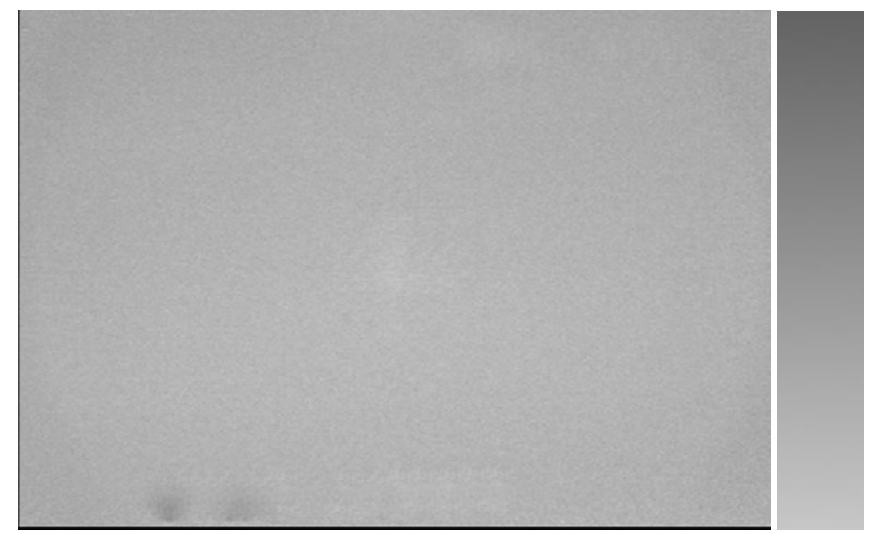

$29.4^{\circ} \mathrm{C}$

$30.6^{\circ} \mathrm{C}$

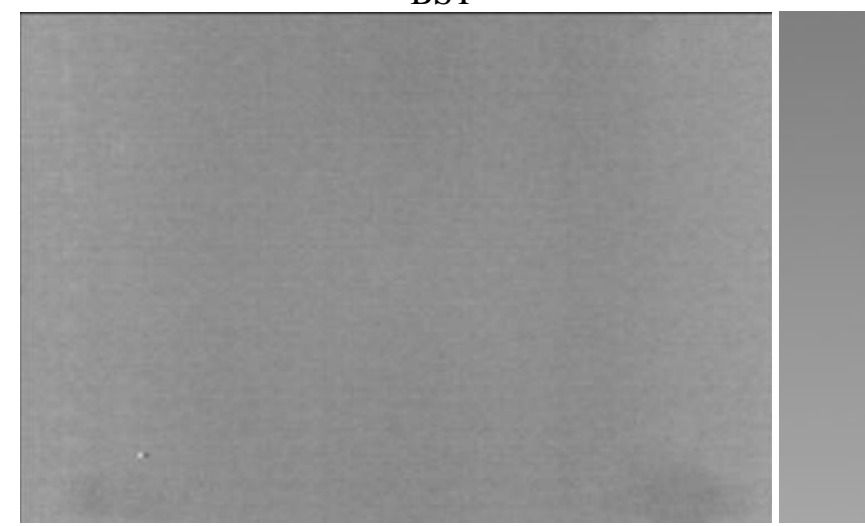

$29.5^{\circ} \mathrm{C}$

$\mathrm{VOx}$

$30.5^{\circ} \mathrm{C}$
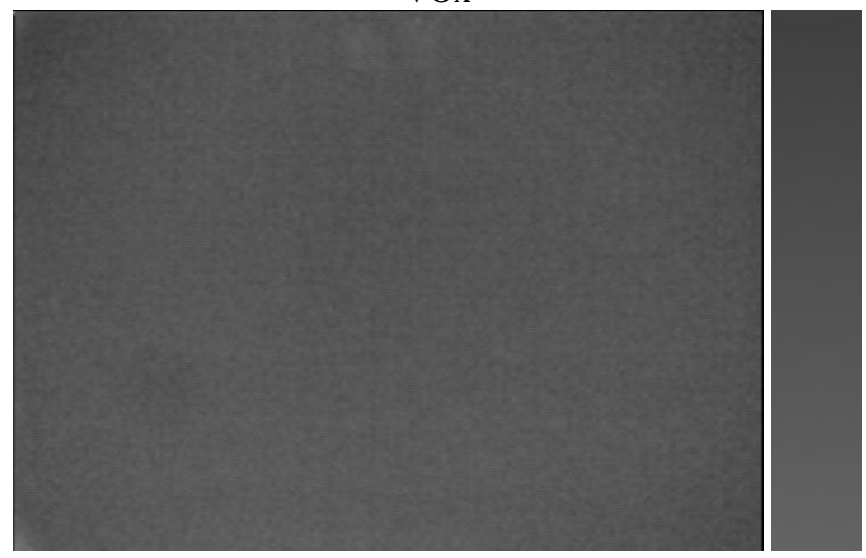

$27.0^{\circ} \mathrm{C}$

$33.0^{\circ} \mathrm{C}$

$\mathrm{ASi}$

Fig. 3. Nonuniformity images of extended area blackbody set at $30^{\circ} \mathrm{C}$ collected from three different TIC detector technologies. Each image shows relative pixel intensities and has a resolution of 640 by 480 pixels. 


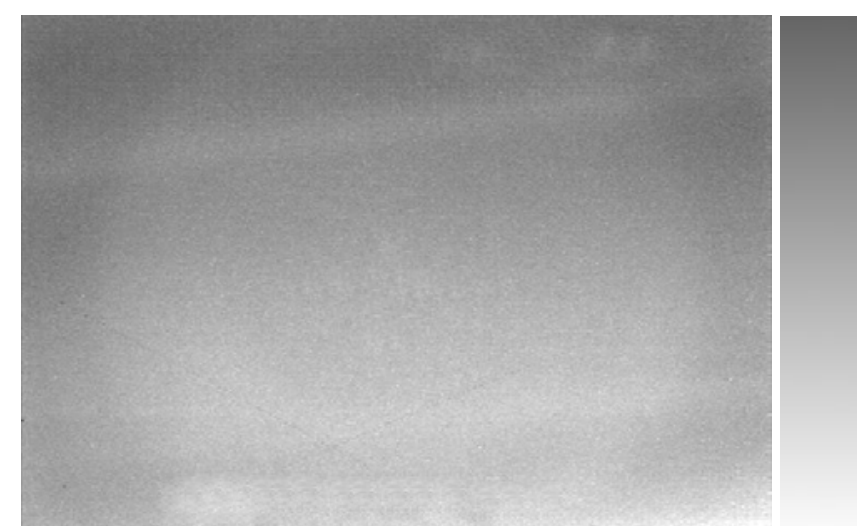

$88.0^{\circ} \mathrm{C}$

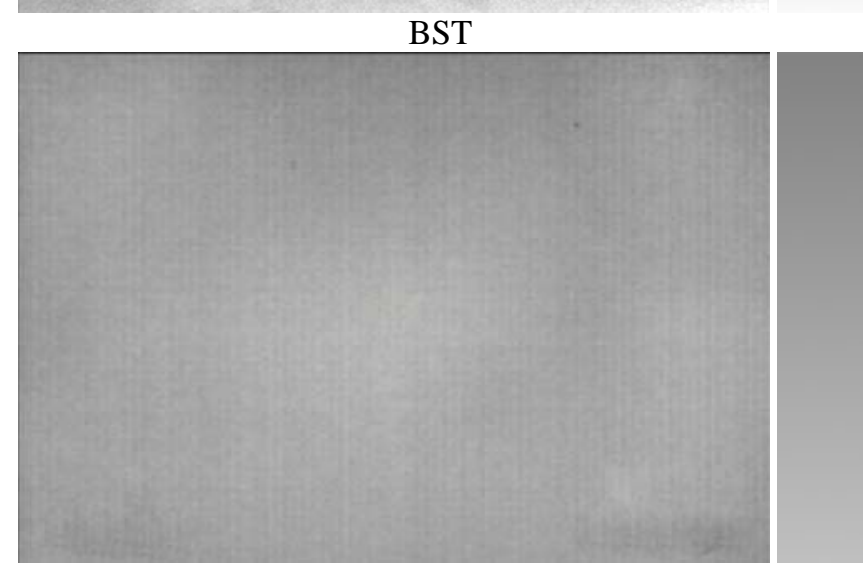

$112.0^{\circ} \mathrm{C}$

$97.0^{\circ} \mathrm{C}$

$103.0^{\circ} \mathrm{C}$

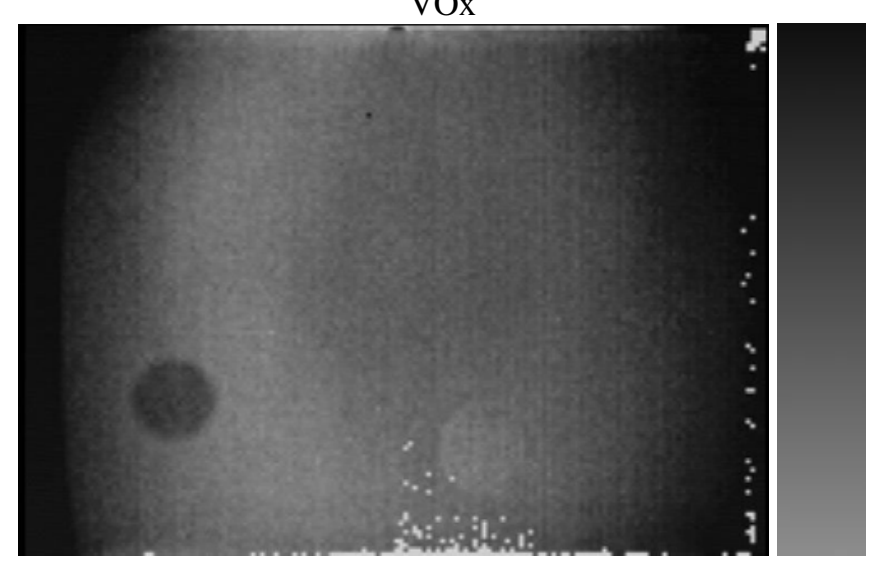

$60.0^{\circ} \mathrm{C}$

$\mathrm{ASi}$

$140.0^{\circ} \mathrm{C}$

Fig. 4. Nonuniformity images of extended area blackbody set at $100^{\circ} \mathrm{C}$ collected from three different TIC detector technologies. Each image shows relative pixel intensities and has a resolution of 640 pixels by 480 pixels. 

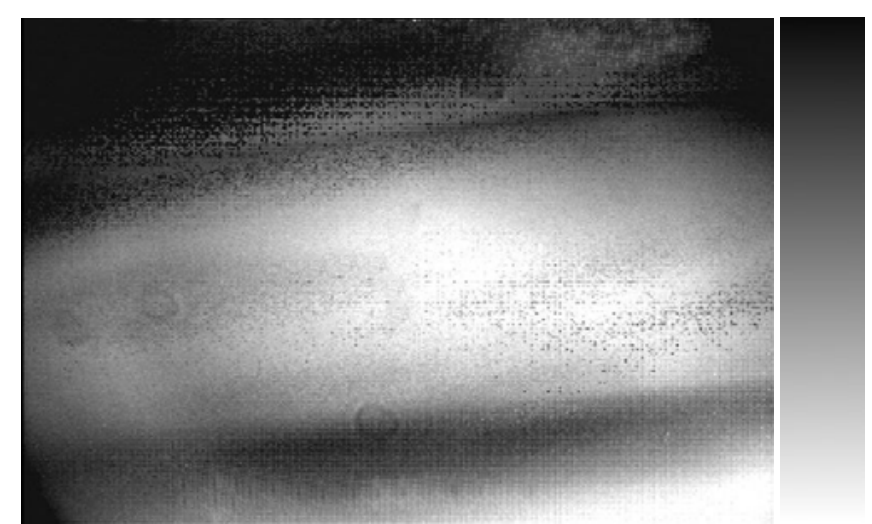

$163.0^{\circ} \mathrm{C}$

BST

$237.0{ }^{\circ} \mathrm{C}$
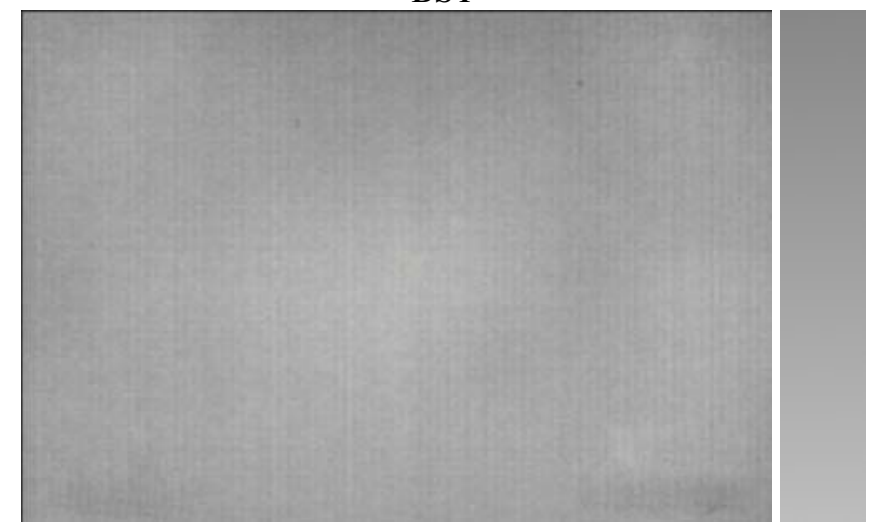

$94.0^{\circ} \mathrm{C}$

$106.0^{\circ} \mathrm{C}$

$\mathrm{VOx}$
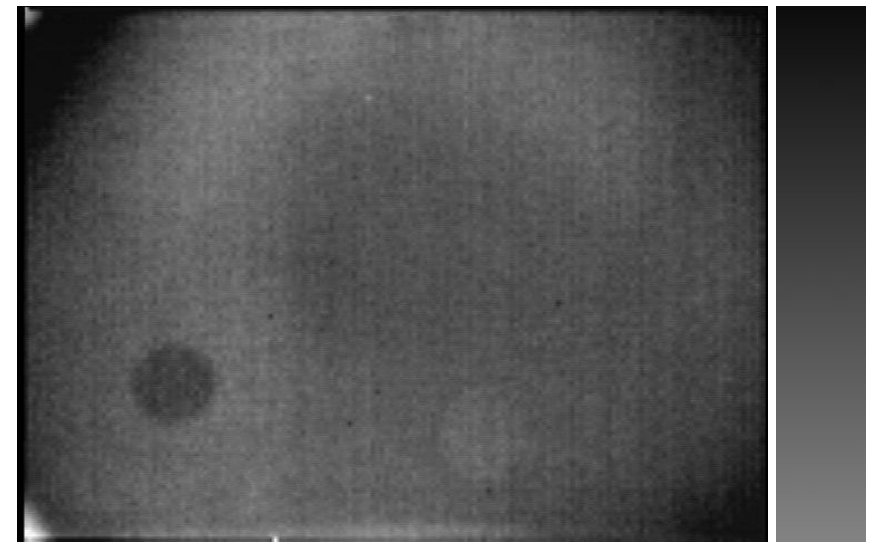

$165.0^{\circ} \mathrm{C}$

$235.0^{\circ} \mathrm{C}$

ASi

Fig.5. Nonuniformity images of extended area blackbody set at $200^{\circ} \mathrm{C}$ collected from three different TIC detector technologies. Each image shows relative pixel intensities and has a resolution of 640 pixels by 480 pixels. 

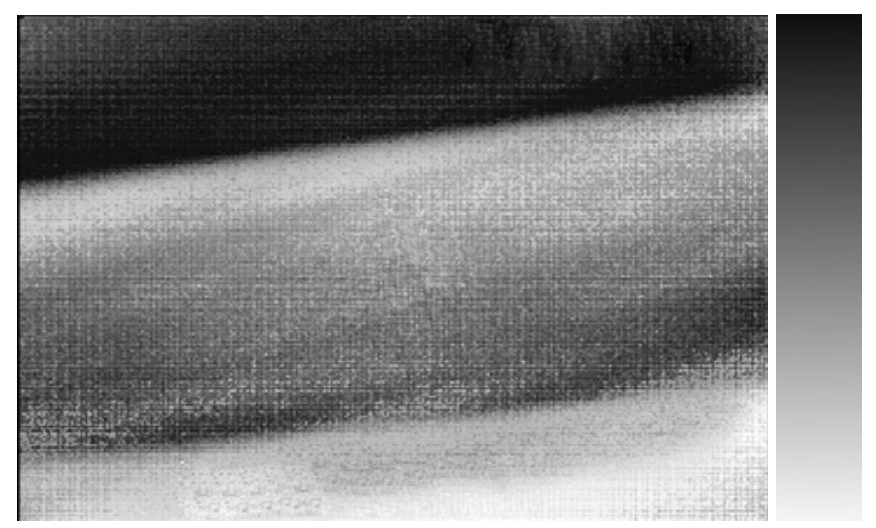

$195.0^{\circ} \mathrm{C}$

BST
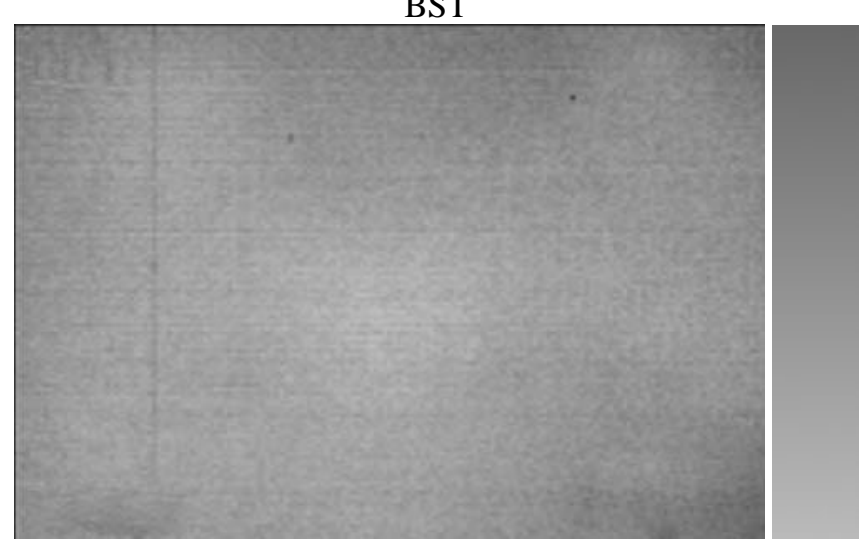

$325.0^{\circ} \mathrm{C}$

$249.0^{\circ} \mathrm{C}$

$271.0^{\circ} \mathrm{C}$
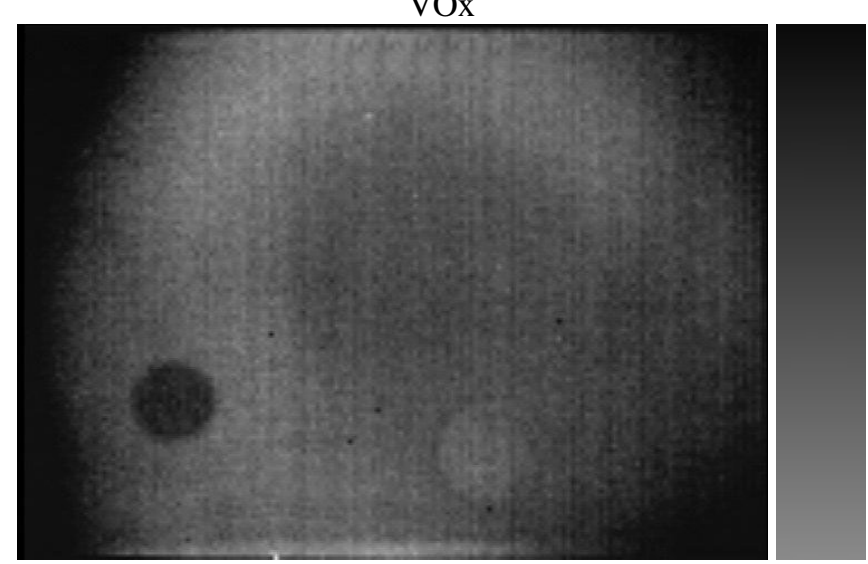

$205.0^{\circ} \mathrm{C}$

$315.0^{\circ} \mathrm{C}$

$\mathrm{ASi}$

Fig. 6. Nonuniformity images of extended area blackbody set at $260^{\circ} \mathrm{C}$ collected from three different TIC detector technologies. Each image shows relative pixel intensities and has a resolution of 640 pixels by 480 pixels. 


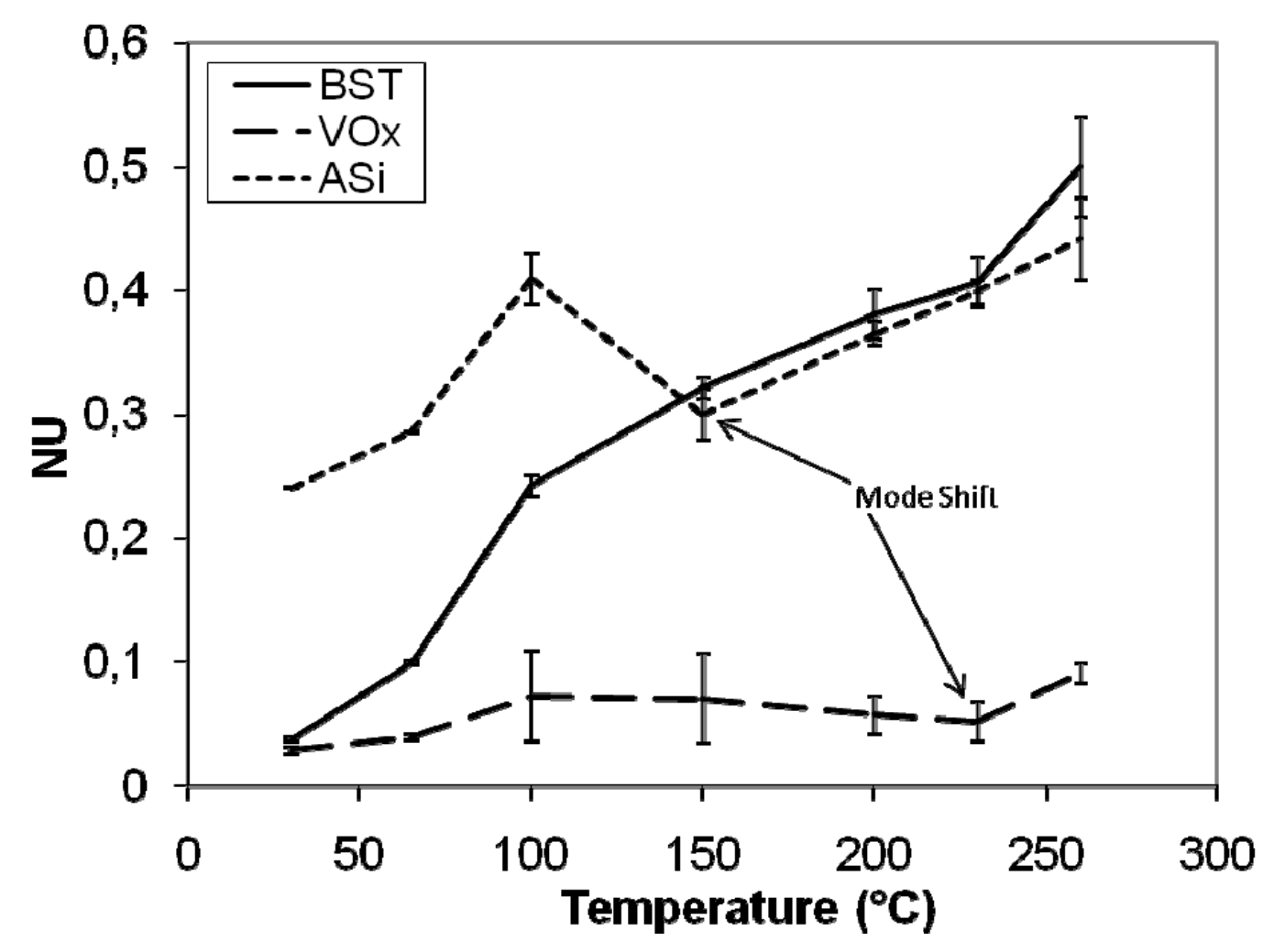

Fig. 7. Quantitative nonuniformity measurements plotted as a function of temperature for each of the cameras tested.

\section{CONCLUSIONS}

This paper presents the development of a practical method for measuring the nonuniformity of TICs used by the fire service. Due to the proprietary nature of each TIC and the wide variety of technologies available, each camera was treated as a 'black box' with identical inputs being given to each TIC and outputs recorded in an identical way. This also precludes the necessity of identifying the specific source of nonuniformities in the TICs, i.e., if the nonuniformity is due to optics, sensor defects, internal processing or another inherent effect of a given TIC, all nonuniformities are treated the same. The primary observations and conclusions of this investigation are as follows:

1. Nonuniformity measurements were conducted with a $178 \mathrm{~mm}$ blackbody placed $5 \mathrm{~cm}$ from each TIC's lens. This puts the blackbody target surface severely out of focus, since the minimum focal length for most of these TICs is on the order of $1 \mathrm{~m}$. It is assumed that the blackbody surface is: a) perfectly uniform, and b) perfectly Lambertian. Under these assumptions, the out-of-focus blackbody target surface is equivalent to using a perfectly uniform surface of infinite extent.

2. The majority of the work involved in testing the TICs was in setting up the test to achieve a uniform field of view and recording the images appropriately. For each test a series of images was recorded in the form of video and the resulting series of digital images was treated statistically to find the standard deviation and mean values of each image to calculate the nonuniformity. The reported nonuniformity value was the average of all of the nonuniformity values from each test, the uncertainty is taken as the standard deviation of the series of nonuniformity values from each test.

3. Examples of the types of nonuniformities observed for each TIC are presented. Even though the type of the nonuniformity is irrelevant to the measurement of the gross nonuniformity, it is instructive for test personnel and users to identify nonuniformities visually without performing the 
statistical analysis necessary to calculate a value for nonuniformity. Some of the possible nonuniformities observed in the TICs include: narcissus, manufacturing residuals, pixel saturation, optical defects, optical misalignment, and other inherent nonuniformities which can not be corrected without inspecting the TIC's internal components.

4. Variation of the nonuniformity value with temperature was presented for each TIC. It is observed that, within the range of temperatures tested, each TIC's nonuniformity increases roughly linearly with the target surface temperature. Non-monotones exist in the $N U$ vs. $T$ plots for some TICs because of their automatic mode shift mechanism, which is designed to decrease the integration time when some percentage of detector pixels become saturated from exposure to overwhelming surface temperatures; this mechanism effectively sets the TIC into a lower thermal sensitivity mode and decreases the nonuniformity of the camera.

5. Differences in the nonuniformity performance between different TICs are indicative of the specific TIC design, not necessarily the detector technology. However, different behaviors are observed for different technologies, one example is the existence of a mode shift for VOx and ASi TICs, which is absent in the BST TIC. Nonuniformity is not the only indicator of TIC image performance and must be considered in conjunction with other image quality metrics such as spatial resolution and thermal sensitivity.

\section{REFERENCES}

[1] NFPA "Standard on Thermal Imagers for the Fire Service;" National Fire Protection Association, NFPA 1801: Quincy, MA, under review.

[2] Holst, G. C., Testing and Evaluation of Infrared Imaging Systems. JCD Publishing, Winter Park, FL, 1998.

[3] Milton, A. F.; Barone, F. R.; Kruer, M. R., (1985) Influence of Nonuniformity on Infrared Focal Plane Array Performance, Optical Engineering 24, (5): 855-862.

[4] Scribner, D. A.; Sarkady, K. A.; Caulfield, J. T.; Kruer, M. R.; Katz, G.; Gridley, C. J. "Nonuniformity correction for staring IR focal plane arrays using scene-based techniques", SPIE, 1990; 1990; pp 224-233.

[5] Scribner, D. A.; Schuler, J. M.; Warren, P.; Howard, J. G.; Druer, M. R. "Image Preprocessing for the Infrared", SPIE, 2000; E. L. Dereniak; R. E. Sampson, (Eds.) SPIE: 2000; pp 222-233.

[6] Green, W. J.; Maurer, D. E. "Merlin Microbolometer Camera Calibration", SPIE, 2001; G. C. Holst, (Ed.) SPIE: 2001; pp 12-17. doi:10.1117/12.439151

[7] Mooney, J. M.; Shepherd, F. D., (1996) Characterizing IR FPA nonuniformity and IR camera spatial noise, Infrared Physics \& Technology 37, (5): 595-606. doi:10.1016/1350-4495(95)00133$\underline{6}$

[8] Donnelly, M. K.; Davis, W. D.; Lawson, J. R.; Selepak, M. S., (2006) Thermal Environment for Electronic Equipment used by First Responders, (NIST Technical Note 1474).

[9] Dinaburg, J. Thesis: Developing Performance Metrics for Thermal Imaging Cameras. University of Maryland, College Park, Maryland, 2007. 